\title{
APPLiCATION OF GAME-BASED LEARNING IN MATLAB USING OBJECT-ORIENTED PROGRAMMING
}

\author{
Karel Perutka \& Michal Vymazal
}
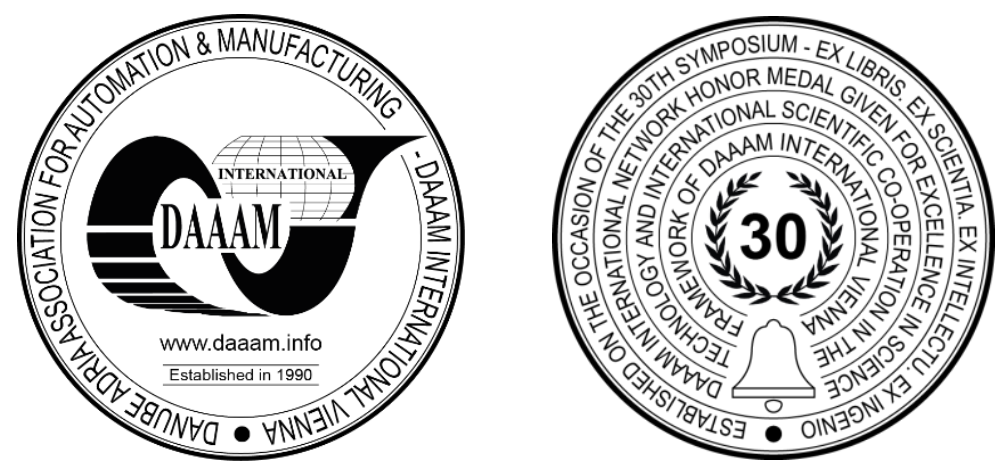

This Publication has to be referred as: Perutka, K[arel] \& Vymazal, M[ichal] (2021). Application of Game-based Learning in MATLAB using Object-oriented Programming, Proceedings of the 32nd DAAAM International Symposium, pp.0022-0032, B. Katalinic (Ed.), Published by DAAAM International, ISBN 978-3-902734-33-4, ISSN 1726-9679, Vienna, Austria

DOI: $10.2507 / 32$ nd.daaam.proceedings.004

\begin{abstract}
This paper focuses on game-based learning and educational digital games. The first theoretical part summarizes the basic concepts and follows the problems with the introduction of game-based learning in practice. The work focuses on gamebased learning as a modern method of education, which is used by many experts from practice and confirms the benefits of its use in teaching. The next part is devoted to specific types of players, then presents the characteristics of digital educational games used in practice and tries to map the use of this method of education in teaching abroad and in the Czech Republic. Furthermore, examples of the use of digital games in teaching and in other industries are presented. The practical part deals with the design of the original game, designed primarily to teach MATLAB, the description of the created application from the user's point of view, and its verification by students during teaching. The game was created using object-oriented programming in MATLAB, which is a very rare and new approach.
\end{abstract}

Keywords: Game-based learning; computer game; education; object-oriented programming; MATLAB.

\section{Introduction}

The issue of game-based learning is currently a frequently discussed phenomenon presented in electronic media [3], [4]. The first technical elements in the process of education and learning include the use of pictures and symbols during lessons followed by a spoken word that complements the printed text. Over time, in the field of education technology development occurs great progress when computers came in the 20th century. It is a time of more significant change, a time of great progress, with books and television being replaced by the Internet and digital games [5], [6].

The advent and constant expansion of digital technology mean a major impact on the whole society [1], [2]. People in Europe, the USA, Japan, and China are most affected by this technology. Digital technology enters several areas in which it fulfills the function of education through play. First, the method of John Amos Comenius can be mentioned as a "school by play" whose main idea is "Teaching is fun", and it is still used today. Today's world, in an environment connected with technique, offers an educational method called "Game-based learning". The concept is possible to conceive as "digital game-based education or digital learning games". This method of education permeates the entire paper. 
The goal of game-based learning is to find out how and where it is possible to incorporate the simpler, more effective, and more interesting method of education involving digital games. Implementation using object-oriented programming is quite a new approach [7], [8], [9]. But it is popular to use games for teaching, there we created nice applications in this field [11], [12], [13]. This paper contains a brief overview of educational games with the potential to educate and also own educational games based on game-based learning [10].

\section{Theoretical background}

\subsection{Definitions}

Game - Basically, there is no clear definition that defines the very concept of the game. Because it is this work focuses on the issue of game-based learning, we will use the definition of one of the most respected experts in this field, Marc Prensky, whose goal is to make the game engaging. According to his publication, the following structural elements serve this purpose:

- Goals

- Rules

- Interactivity

- Entertainment

- Adaptability

- Winning states

- Outputs and feedback

- Conflict/rivalry/challenge/objection

- Interaction

- Problem-solving

- Representation and story

From the above, it is clear that there is no uniform definition of the game, not even within one scientific field. Because with many definitions, the game is characterized by key elements, we assume that they are necessary for its functioning. However, not every game has to identify with all key elements. Key elements are:

- Rivalry - The goal is to achieve a better result than others can on students act on two factors, motivational and demotivating. There can also be rivalry affected by competition

- Illusion - The existence of an environment that can be explored, commonly used in the simulation.

- Interaction - is an important element of education, it helps students better understand the topic substance, group teaching is recommended, in the form of discussion or counseling, (action changes the state of the game and triggers feedback

- People - more people are recommended (students, teachers)

- Challenge - tasks require effort and are not very trivial, so they become challenging for him and difficult

- Rules - should be understood as recommendations rather than regulations, and thus lead the student to successful education

- Exploration - The existence of an environment that can be explored

- Security - this is a quiet place, set for the learning process and verification knowledge of students.

- Results - specific results are important for evaluation (e.g. scoring)

- Goals - explicit goals and objectives.

- Research - is important, looking for new interesting information, offers other questions, researches the more detailed topic

Digital game - The word digital means the use of some kind of electronic device that can, however, we don't have to be connected to the internet. These devices include, for example, laptops, pocket gaming consoles, classic desktop computers, mobile phones, music players, etc. These are actually about a device with a microchip that allows you to play the game.

Video game - Some people have no problem imagining a digital game under this name. It can be said that They are right because video games are the equivalent of a digital game, but the display of the game is the screen that is required. The main purpose of video games is to entertain the players.

Computer game - Computer games are run on a computer or laptop. It is an interactive program that allows you to communicate with the user using sounds, 2D and 3D graphics, and text. The form of tension and fun fills and diversifies the free time of all players. 


\subsection{Types of players}

Bartl's taxonomy - Richard Bartle (1960) is a British writer, professor, and researcher in the online gaming industry according to the structure, there are multiplayer games. For example, in collaboration with Roy Trubshaw, was created game MUD (Multi-User-Dungeon) was. It is a game based on the virtual world, specifically on text. Bartl took advantage of the game to create a survey to find out what games players enjoy, as different players may not have the fun same game. According to the behavior of players in the community (Multi-User-Dungeon), it was found that players are divided into four categories: Achiever, explorer, socializer, killer [10].

Achiever - The main goal of the player is to get as many awards or rewards in the game world as possible, for example, badges, points, money, levels, and more. The main motivation for players is to achieve goals and win. He adapts the tactics to obtain as many material things as possible. The player is accompanied constantly by the idea of comparing with other players.

Explorer - This type of player focuses on individual elements of the game world. First, he maps out what rules the virtual world works with. Then he looks for shortcomings. The usual characteristic of A player of this type is that he admires secret places and other unexplored locations. It does not accept restrictions games and trying to overcome them. Once he finds nothing new, the game becomes unattractive for him.

Socializer - This type of player is primarily interested in socializing, so communication with people is at stake most important to him. The player strives to create a strong relationship in the sharing community thoughts, opinions, stories. The game itself, for him, is not so important. Games for one player only lays when this game attracts a lot of attention from the surroundings. Just because to talk to other players about the game. His biggest weakness is the fear of rejection.

Killer - A killer is the type of player he kills to defeat an opponent. The player can play games from sports to action, the most important element is the opponent. From the psychological point of view, this type of person exploits his aggression by bringing it into play.

\subsection{Game-based learning}

The term game-based learning can be translated as "learning through digital games or education based on digital games." It is a combination of digital games with form education. Today's students are accustomed to digital technology from an early age, and therefore they want to combine it with teaching. The primary goal and premise of game-based learning (GBL) is teaching that makes students more enjoyable and based on which it is possible to achieve the same or even better results than the classic form of education, which is already boring for students.

Certain procedures and teaching methods have been provided for the older generation of students.

Some teachers still think that these methods could work today. This idea has already been refuted. "Today's students are no longer the people for whom our education was system designed". There is a problem for both teachers and students. The problem of teachers is engaged with their teaching method and effectively educate students. Students can't concentrate and usually tend to be bored and inattentive. Therefore, it is appropriate to change the teaching, to match current education. By introducing a more interesting and fun form of teaching we achieve educational games.

Educational games are seen as the opposite of fun games, otherwise translated as "serious games". Sarou de Freitas defines educational games as: "Applications using characteristics of video games and computer games to create a catchy learning experience to transfer of identified learning objectives, outcomes, and experiences ". There is still debate among educators on the issue of digital games in the field of education, so-called digital game-based learning.

The game with educational content works as a means of education mainly in fulfilling these reasons:

- By including the content of education in the game, some obligation arises that may happen interesting for students, aversion to learning.

- Depending on the educational goals, it is possible to apply different methods of the interactive educational process.

- The grouping of the interactive educational process and the integration of the educational one content into the context of the game. It can be achieved in several ways, but the best solution will follow according to the needs of the teacher and the student.

A game that meets these requirements can become completely ineffective, if not the educational content does not correspond to the abilities and interests of the student.

Practical use - Digital games serve not only as a very important and indispensable element in teaching but as well as preparation for various crises. Examples are natural disasters or terrorist attacks requiring rapid decision-making under time pressure. Their use can also be used in training for driving cars, controlling aircraft, to create models of solutions to problems in different areas, or serve as a tool for team building and team training cooperation that promotes goal-oriented coherence. 
It also allows providing information on social networks, and especially its importance as a team is needed within companies and in their cooperation. Digital educational games are a success used in many sectors, such as insurance, law, medicine, pharmaceutical industries, banking, etc. Subsequently, I deal with these in more detail industries in which game-based learning is used - education, employment, military, economy.

\section{Selected computer games created in MATLAB}

In the following chapter, we will find examples of games in MATLAB. The criterion for choosing these games was the fact to what extent they were fun. How they improve logical thinking and motivation to the game. All of the following games can be found on the server of MathWorks.

\subsection{Chess}

This is one of the oldest games and the first mentions coming from the western world are dated to the 6th century. The game comes from India, the date of origin cannot be determined exactly. Los Almos is the first program developed to play chess and dates back to 1956. According to available Paul Stein and Mark Well for the MANIAC I computer was to take part in the development of the information. The basis of the game is a chessboard measuring $8 \times 8$. On the board, you can see that individual the squares of the chessboard alternate between the two colours for better orientation. For complete clarity as well used to name individual rows and columns. The player can play for either a white or black group of figures. The first version of Chess (Chess Master) in MATLAB, programmed by Brian Moore was created in 2014.

\subsection{8}

The original game was programmed in JavaScript. This is not a particularly old game, the game dates back to 2014 and the author of the game was Gabriele Ciruili from Italy. The playing field is about the size of a $4 \times 4$ grid (16 squares). When using the arrows, the boxes move according to the selected direction. The game works on the principle of connecting two cells, with the same number. This way we get to the number 2048. This is a game created in 2014 in MATLAB by an employee of MathWorks, nicknamed Jiro.

\subsection{MATLABTETRIS}

Tetris is the most successful computer game in modern history. This is a game programmed in MATLAB, from 1985. The main author of this game is Russian scientist Alexei Pažitov. As the name suggests, each shape consists of four squares. Everybody shape falls from the top down and our goal is to place it in the lower part of the playing area so that it will be filled in its entirety line so that it can then be deleted. The game automatically increases the difficulty the longer it is played time, thereby increasing the speed of the falling body. The game itself is controlled with the arrow keys. The up arrow has the function of turning the page and the down arrow has the function of setting body fall velocity. In the game environment, the individual shapes that follow the existing ones are displayed. It is too available information table, in which it is stated: number of points obtained, current level and the number of rows deleted. Matt Fig reprogrammed the game into MATLAB, releasing a total of two versions in the same year 2012.

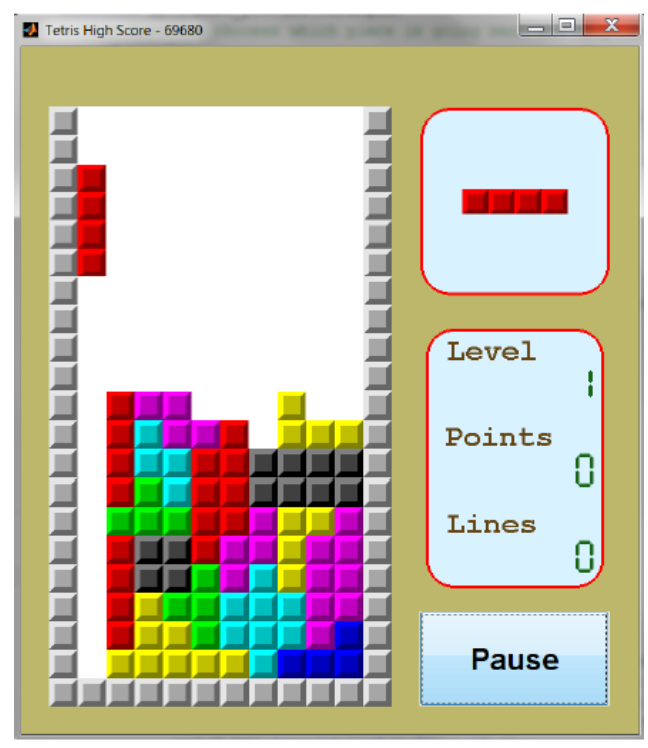

Fig. 1. MATLABTETRIS main window 


\subsection{Pac-Man}

Toru Iwatani is the author of this famous computer game, which he named Pac-Man. Toru Iwatani began to develop it because there was no other similar game on the market. Most games in the given tend to come more from the action game industry. The aim of the game is for Pacman to collect all the balls. But a player who plays for Pacman must be careful not to be caught by ghosts. There is also the possibility of temporary control over ghosts. To gain this power, the player will have to eat a ball that is larger than the other. This would put Pacman in a phase where he could hunt ghosts and get some for it points, but this moment is usually very short. Once Pacman collects all the balls, gets to the next level, where the ghosts gain higher speeds. Athanasios Kehagias rewrote this game to MATLAB, working specifically with the version R2016a. Now the current version is from 2017.

\section{A new application of game-based learning in MATLAB}

TBU Quiz is a new 2D educational game focused primarily on testing your knowledge players. In general, he can verify knowledge in various areas, because it is enough to exchange question and answer database files. And all in a fun way. The game is programmed in general. It is currently focused on knowledge of MATLAB, but in the future, it can be used to test any other knowledge. The list of questions for the quiz is not strictly limited to MATLAB and is freely distributable.

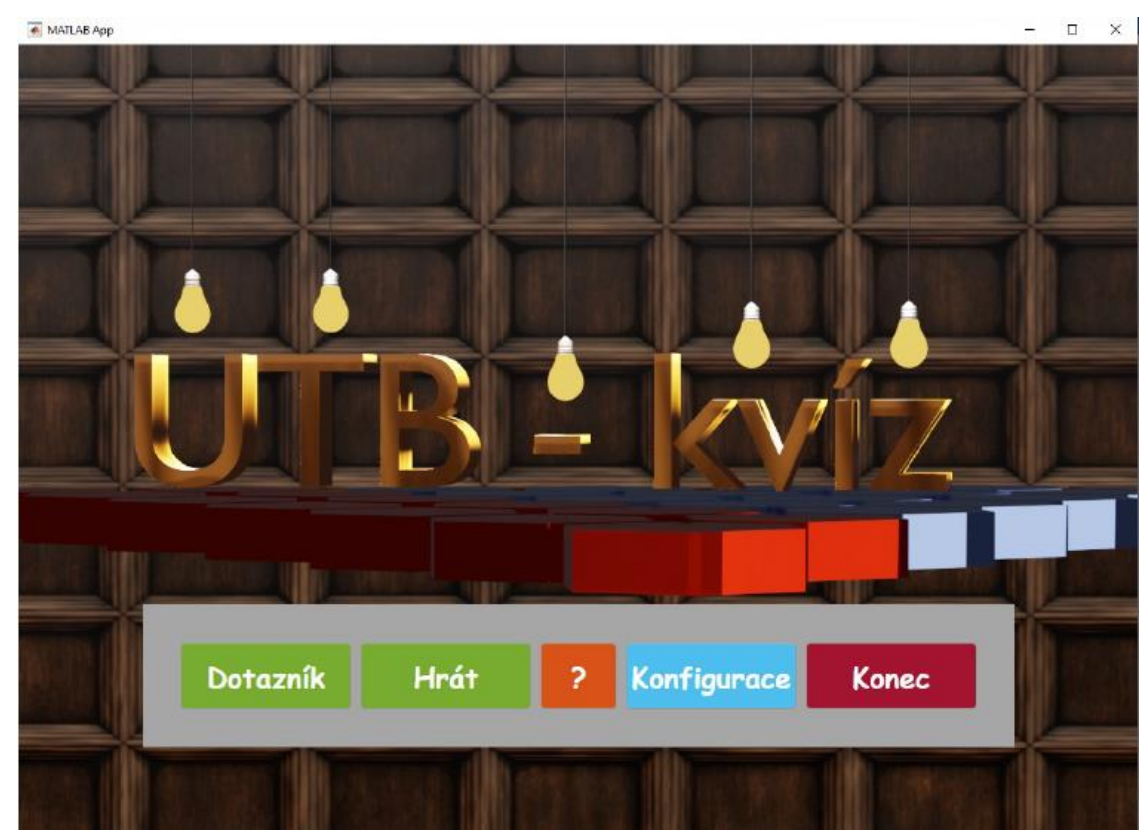

Fig. 2. Opening window of new game

\subsection{Game breakdown}

The game is divided into a total of four parts, which are configuration, help, questionnaire, and the quiz itself. Each part is designed to be fully managed and controlled by a graphical interface created using MATLAB. Players and any administrators thus are not burdened with the knowledge of the structures of the files used and their interconnection. Everything is possible conveniently set up with an intuitive interface.

\subsection{Configuration}

Most games, whether simple or complex, allow a variety of settings a potential player can adjust the environment according to their preferences. This is not the case with TBU Quiz either otherwise. This is not a configuration in the true sense of the word, but rather a simpler derivative. It does not allow any customization of the interface, but only the basic configuration. Specifically, it goes to manages the quiz and questionnaire questions on which the whole game is based. That's why it's their management is an integral part of the game.

\subsection{Help}

Help is another invaluable helper in every right game. That's why it's not missing either here. The game itself is quite intuitive, so it wouldn't even need help, but only for the completeness of the solution deserves it. 


\subsection{Questionnaire}

The questionnaire part is the only parallel to the main playing part. You could say it works about a minigame designed to analyze the type of respondent, in this case, the player, based on provided answers. It may seem that such a questionnaire does not completely fit into the strategy quiz games, especially if it focuses on knowledge of MATLAB syntax and semantics. On the other hand, it is very valuable information that the game provides, without any special knowledge. Interestingly, there is no wrong answer. Each so can shape the character of the player.

\subsection{Quiz}

The quiz is the main part of the application. The part that every player looks forward to when running any games. Because it is a variation on a similar, well-known television quiz that it is intended for two players, and here it is no different. As with the TV quiz, it is the alpha and omega everything a list of predefined questions hidden behind the field that the player chooses. Unlike the TV version of the game offers a list of potentially correct answers, when correct is always only one. The second difference is the way individual fields are assigned to players. In the TV quiz, the selected field, to which the player answers incorrectly, is neutralized. TBU Quiz as part of simplification and optimization assigns it to opponents. The third and also the last main difference is the evaluation of both players after completing the quiz. The game at its end evaluates both the knowledge of the winner and the opponent. They will both find out how they are doing with their knowledge.

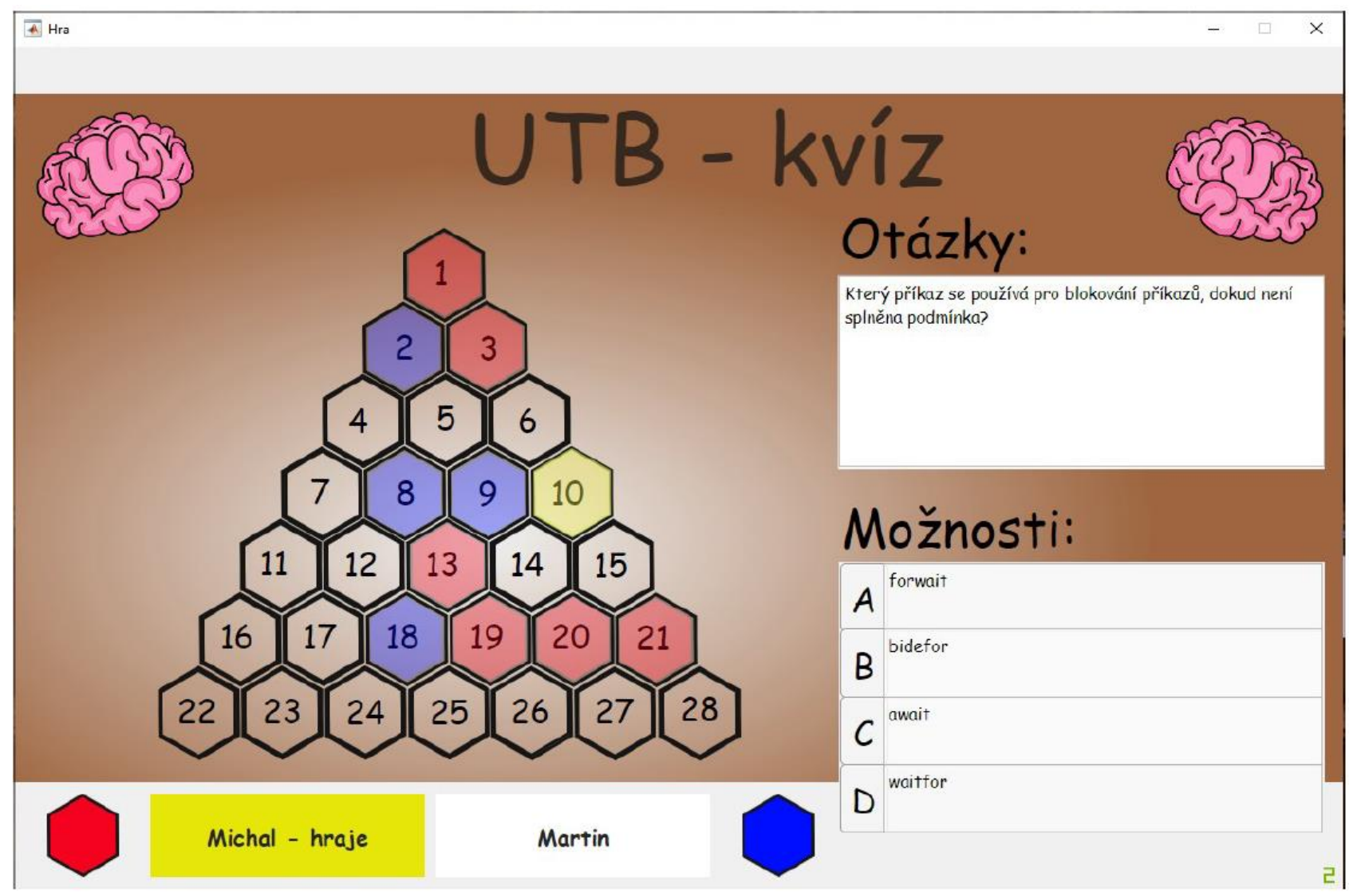

Fig. 3. The main window of new game

\subsection{Quiz rules}

The quiz is intended for two players and is not limited by any number of rounds or time limits. The game ends when one of the players manages to connect all three sides with their fields playing pyramids. The fields defining the winning path through the pyramid are marked for clarity in light green colour. The game statistics of both players are displayed. Each round always determines only one active player. They take turns. Active the player must always choose one free field hiding the question. After making a selection, it is displayed the question and the active player must answer it by clicking on one of the buttons "A", "B", "C" or "D" that precedes the answer. If the answer is evaluated as correct, the field is assigned to the active player. Otherwise, the field is assigned to the opponent. In both cases, however, the field cannot be re-selected during the game. 


\subsection{Data storage}

The game itself, in essence, places only minimal demands on any data storage, possibly the need to use a database system. It requires only two specific files that store information about questionnaires and quiz questions. To store this data, just a simple file system will suffice. Both of these files must be in the directory Config, which is located relatively at the same level as all other files' applications.

- Questionnaires.xml is a file data structure that stores all questions and the relevant answers used in the questionnaire. The file is for easy editing in xml. Despite this fact, it is not recommended to edit it outside the configuration panel itself application.

- Questions.xml is a file data structure that stores all questions and relevant answers used in the quiz. The file is for easy editing in $\mathrm{xml}$ format. Despite this, however, it is not recommended to edit it outside the configuration panel of the custom application.

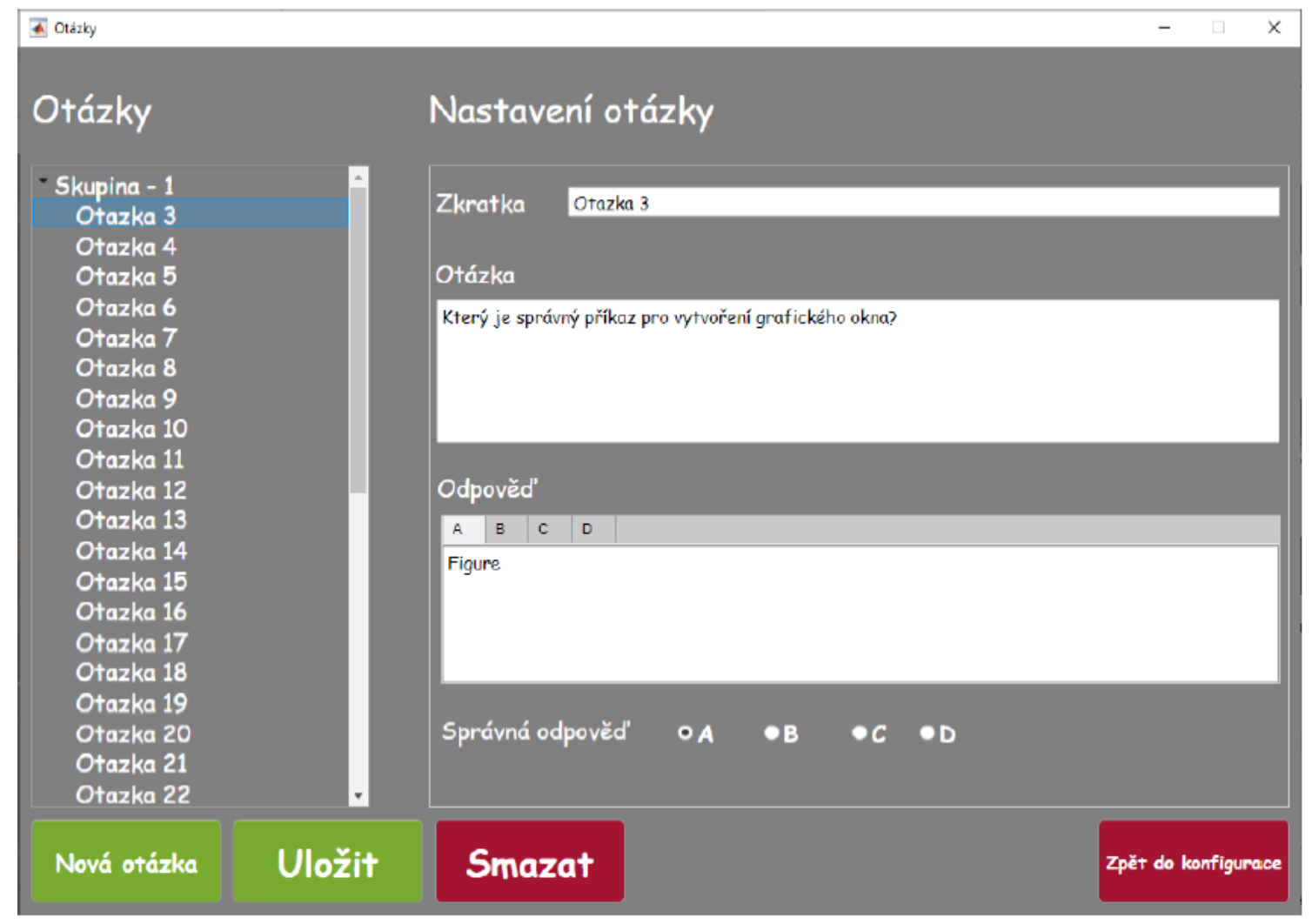

Fig. 4. The window of quiz data configurator

\subsection{Main algorithm}

Matlab brings with it various limitations in the development of form applications. For an application containing only one form, there is no need to deal with any additional complexities and eventuality. However, in case there is a need to use more than one form on the fly application, a few major obstacles need to be addressed. An example is a modal form, to ensure the risk of closing a form other than the one currently open. Next An issue that needs to be addressed in a multiform application is passing parameters between forms. Last but not least, it is also a challenge to deal with various generic lists, such example, queues, dictionaries, or data sheets only. The main application form is MenuWindow.mlapp. It is only after its completion that it ends the whole application. For this purpose, the game uses two principles that ensure the impossibility of his closing before all other forms are closed. The first principle is the implementation of top bridge technology.

This informs the operating system of the highest display priority the form so marked. Any other form is then displayed after this, namely including forms from other applications. But this alone is not enough for the desired behaviour, because it is still possible to close another form than currently displayed, thus getting the application into a non-deterministic state. Therefore, the application still internally implements an internal state in the forms, which controls whether it is possible to close or not. The state is represented by a variable containing the logical value 0 or 1 . If any other form is displayed, this variable is set to a logical value of 0 , making it impossible to close the form from which the new modal was displayed form. In its close callback, it checks the status of the variable and if it is set to logical 0 , cancels it and does not close. 


\subsection{Algorithm evaluating pyramid questions}

One of the main algorithms of the whole application is undoubtedly the evaluation algorithm game pyramid issues. It runs in each round of the game quiz. The algorithm begins by switching the current player to an opponent. As for the first round, set to the current player always red. It continues by loading user input in the form of the selected field. If when the field is occupied, it returns to the game field selection. Otherwise, it loads the question also answer and display them on the board. In the next step, it reloads the user input in the form of selected answers. He has to evaluate it. If the answer is correct, it assigns the selected field active player. Otherwise, it is assigned by the opponent. In both cases, however, it continues by evaluating the completeness of the user's path. And be an active player if it is the right one answer or the opponent in the case of an incorrect answer. The player's path is evaluated using graph theory. Specifically, the widththrough algorithm. This is also the case of a signature algorithm, which is also the most complex. If the path of the player, if any opponent is incomplete and does not connect all three sides of the pyramid, the algorithm returns to the beginning. Here the player switches to the opponent. The algorithm is executed until one of the players reaches the completeness of the journey and thus wins.

\subsection{Algorithm evaluating the completeness of the path}

TBU Quiz uses graph theory to evaluate a player's path in the game pyramid. Specifically, searching the graph in width. This algorithm requires a queue at the beginning of processed items. Matlab offers AsyncBuffer for this purpose. AsyncBuffer allows you to read elements exactly in the order in which they were written to it, which is a great advantage in processing. After creating the queue, the algorithm will add it directly to the field currently being processed. It also creates an auxiliary list for the fields that have already been processed need to remember, otherwise there would be a loop. Here Matlab provides a function containers.Map, which allows the definition of the dictionary structure.

When the algorithm has a queue and an auxiliary list ready, it starts a cyclic pass the element queue, which it executes as long as the queue contains something. For each individual, the field within the cycle reads all neighbouring ones and also tests them cyclically. If given the field belongs to the player and is not in the help list, it adds it to the queue for processing and continues to the next until he passes all the neighbours. The moment the queue is empty, he checks the algorithm the last condition that is required to end the game by winning, and that is connecting all three sides of the game pyramid. This part is already simple. Here the algorithm only tests whether the auxiliary queue of processed elements always contains at least one field on each side of the game pyramid and if so, ends the game by winning the player. Otherwise, the game continues for another round.

\section{Application verification}

\subsection{Self-testing}

Before it is possible to pass the application containing the game to the agreed testers, it must go through its testing process. If this were not the case, possible errors would delay testers and distracted them from the very essence of game testing. The result of such testing should not be a list of bugs, but suggestions for improving or modifying existing algorithms.

Internal testing takes place in three steps. The first is to test the baseline scenarios application control. The second is playability testing according to the documentation and the third is random, one could say that even a chaotic click through the whole application. In general, development should never be tested by a programmer. Or more precisely, to test certainly had, but such output can not be considered as a proven functionality, because the programmer knows exactly how he wrote and is affected by this fact during testing. He doesn't always succeed reveal everything. For this reason, the game will go through both internal testing and pre-testing agreed on testers.

\subsection{Testing baseline scenarios}

Testing basic scenarios deals with the issue of basic application control procedures. It focuses mainly on the detection of shortcomings and unfinished business resulting from various additional modifications to algorithms that cause the application to crash unexpectedly and untreated.

Thanks to this testing, it was possible to detect semantic indexing errors of various kinds of collections that are used in the application. The first versions of the game have suffered from this issue since they are built on collections and cycles that go through them using indexes. Generally speaking, that the custom is to index the collection from the number 0 . Thus, the first element in the collection is at index 0. There are two different approaches in MATLAB. One is classically based on indexing from 0 , the second, on the contrary, is from 1 . And that was the source of many problems in the early development of this game. Get used to it Take a dual approach and in each specific case, thinking which one to choose was enough demanding, and this generated a lot of unpredictable situations. An example may be a vector containing randomly shuffled indexes of quiz questions. Such vectors are indexed from 1. But that's not all. If it is cyclical, it is necessary to think even that the vector ends with a length index and is therefore not reduced by one. 
The opposite approach but provides, for example, collections that publish individual XML child elements. This collection is indexed from 0 , so its last element is at the specified index the length of the collection reduced by one. Another problem resulting from this testing was in the object access to data. The program itself contains several classes, where their instances are used in different places application. The class itself is a very useful thing. But the problem arises when it is necessary for its instance, not only to keep the data static but also dynamic. That is if the class must also allow data to change. Specifically, the GamePlayer class stores statistics for a given player. These change after each round that the player plays. And that was exactly the problem. The values of the statistics appear when the instance is created sets the class to 0 . Then, in each player's round, the value 1 is added to the specific one object properties. However, the changed value lasted only in the context of the method in which it was changed. Even if the object was stored in a global variable. As if accessing a variable made local copies. This scandal was removed by inheriting the class from an ancestor named handle.

\subsection{Gameplay testing}

When the application is debugged for unexpected crashes, it is necessary to proceed with testing game algorithms. These were written almost flawlessly. The only problem was the presentation of game statistics of individual players. The achieved percentages were displayed in exponential shapes, or formatted to a lot of decimal places. This problem was more complicated than it may seem. The data is written to the form using the sprintf method. She takes it as parameters a formatted string and an array of values to print in the string. As a solution to a problem, the use of the Round function was offered. But it did not work as expected and the value was still did she misspelled. The solution was provided by changing the format string itself. Originally the value is printed using the wildcard \% $\mathrm{d}$ to be used for integer values. New the $\% 0.2 \mathrm{f}$ symbol is used, which ensures correct formatting to only two decimal places.

\subsection{Final random testing}

Random testing includes a random element in the process. So it tries to get the application to a non-deterministic state by simply randomly clicking on any buttons that the application offers. Usually, this activity is suitable for a person who does not know the application at all and does not know what he is allowed to do and what he is not allowed to click on. By this testing, as in the previous section, more or less only one problem was revealed. But in more places. This problem was the absence of code addressing the logic of the dialog form. More precisely, some forms did not contain the DialogResult property, so they could not be responded to from forms superiors. But that was a problem that caused the game not to be turned off in any way since she did not allow her termination. She was still waiting for the result of the dialogue. The problem was fixed quite simply. The affected forms provided the implementation of the required interface and everything began to work as expected.

\section{Evaluation}

This chapter evaluates what exists, what is new in this paper, and the plans for the future. There exist games in MATLAB and applications of game-based learning written in different programming languages than MATLAB, mostly in western European countries and the United States. But there are only a few of them created or used in the Czech Republic. The same is with the object-oriented programming applications in MATLAB, there is a minimum of them worldwide. This paper combines object-oriented programming in MATLAB and game-based learning with implementation in testing the knowledge of MATLAB, which is a new approach. In the future, a database of questions and answers will be enlarged together with the implementation of another taught subject, discrete control.

\section{Limitations and plans}

Although the paper has reached its aims, there were some limitations. Because the created application is new, it was verified only by a small group of students who were attending the classes. Therefore, it is necessary to use it for some years to generalize the result and to find what to improve and enlarge. Future work is divided into several parts. Firstly, it is necessary to use the materials for more years in purpose to evaluate them from the users' point of view. Lately, there will be realized necessary correction based on the experience with these materials.

\section{Conclusion}

The paper dealt with implementing an important method of education based on digital games. Many authors of professional articles are convinced of the use of games not only directly intended for teaching but also games with quality and useful educational goals, i.e. commercial games. Although it does not exist a unified interpretation of a digital game with educational value, according to a literary survey, several experts agree that sophisticated digital games bring players faster decision-making, develop logical and strategic thinking, and while maintaining thoughtful educational content serves as a full-fledged educational tool. 
The current generation has a huge advantage in using digital educational games and education, as it has an innate ability to take an interest in digital technology and discover the whole digital world. The introduction of game-based learning in schools, in companies, and in other collective activities contributes to maintaining mutual relations and stronger teamwork.

Examples of game-based learning research prove that the world is on this topic. Many professionals have been dedicated to education for many years. Based on the statistics discussed above in comparison with the world, game-based learning is not so widespread in the Czech Republic. But it is already to see a growing interest in a method of education based on digital games. The potential for teaching is huge. This medium can be used to teach various subjects. Scientists and teachers almost agree that games work positively, but at the same time, it is necessary to give a big one and to be careful not to create a gambling addiction to the player, in which about one is seen as the main negative influence.

The current trend is the technology that pupils and students cannot avoid. After leaving school, whether at work or in everyday life, will need and use it. The theme of game-based learning is very likely to appear in a large number of games focused on learning through play, thus realizing a fun form of learning for as many as possible students.

These procedures are implemented in the designed educational game TBU-quiz in MATLAB. This game is for two players. Its task is to connect the cells that are arranged in a pyramid shape. Beneath each cell is a randomly generated question with four answer options. The questions are focused on the MATLAB program. Specifically, it is about three areas of questions. Each topic is represented by a specific cell. To whom first manages to unite all three sides of the pyramid, wins. The resulting window offers a percentage success of players in individual categories. The goal is to use this game for teaching or dissemination of knowledge.

To sum up, in general, what was the problem? The problem was to implement the modern technique of education in the field of MATLAB teaching. How was it solved? It was solved by creating the game using object-oriented programming in MATLAB. What are the solutions? The solution is the created application in MATLAB as teaching What are plans? In the future, we will focus on the enlargement of questions and solution databases.

\section{References}

[1] Sugahara, S. \& Cilloni, A. (2021). Mediation effect of students' perception of accounting on the relationship between game-based learning and learning approaches. Journal of Accounting Education, 56, 100730

[2] Dudzinski, M.; Rozgic, M. \& Stiemer, M. (2018). oFEM: An object oriented finite element package for Matlab. Applied Mathematics and Computation, 334, 117-140

[3] Xu, Y.; Lau, Y.; Cheng, L. J. \& Lau, S. T. (2021). Learning experiences of game-based educational intervention in nursing students: A systematic mixed-studies review. Nurse Education Today, 107, 105139

[4] Kao, Ch.-W. (2020). The effect of a digital game-based learning task on the acquisition of the English Article System. System, 95, 102373

[5] Greipl, S.; Klein, E.; Lindstedt, A.; Kiili, K.; Moeller, K.; Karnath, H.-O.; Bahnmueller, J.; Bloechle, J. \& Ninaus M. (2021). When the brain comes into play: Neurofunctional correlates of emotions and reward in game-based learning, Computers in Human Behavior, 125, 106949

[6] Krath, J.; Schürmann, L. \& von Korflesch, H. F. O. (2021). Revealing the theoretical basis of gamification: A systematic review and analysis of theory in research on gamification, serious games and game-based learning, Computers in Human Behavior, 125, 106963

[7] Richard, B.; Rastiello, G.; Giry, C.; Riccardi, F.; Paredes, R.; Zafati, E., Kakarla, S. \& Lejouad, Ch. (2019). CastLab: an object-oriented finite element toolbox within the Matlab environment for educational and research purposes in computational solid mechanics, 128, 136-151

[8] Berger, R.; Bruns, M.; Ehrmann, A., Haldar, A., Häfele, J.; Hofmeister, B.; Hübler, C. \& Rolfes, R. (2021). EngiO - Object-oriented framework for engineering optimization, Advances in Engineering Software, 153, 102959

[9] Noroozi, O.; Dehghanzadeh, H. \& Talaee, E. (2020). Entertainment Computing, 35, 100369

[10] Vymazal, M. (2021). Application of Game-based Learning in MATLAB, Bachelor Thesis, Faculty of Applied Informatics, Tomas Bata University in Zlin, Zlin, Czech Republic

[11] Karuovic, D.; Radosav, D. \& Glusac, D. (2010). Educational Game Model for Pre-School Children, Chapter 12 in DAAAM International Scientific Book 2010, pp. 107-116, B. Katalinic (Ed.), Published by DAAAM International, ISBN 978-3-901509-74-2, ISSN 1726- 9687, Vienna, Austria DOI: 10.2507/daaam.scibook.2010.12

[12] Polcar, J.; Gregor, M/; Horejsi, P. \& Kopecek, P. (2016). Methodology for Designing Virtual Reality Applications, Proceedings of the 26th DAAAM International Symposium, pp.0768-0774, B. Katalinic (Ed.), Published by DAAAM International, ISBN 978-3-902734-07-5, ISSN 1726-9679, Vienna, Austria DOI:10.2507/26th.daaam.proceedings.107

[13] Musalek, M. \& Adamek, M. (2020). The Use of Virtual Reality Games as a Tool to Overcome Acrophobia, Proceedings of the 31st DAAAM International Symposium, pp.0485-0489, B. Katalinic (Ed.), Published by DAAAM International, ISBN 978-3-902734-29-7, ISSN 1726-9679, Vienna, Austria DOI: 10.2507/31st.daaam.proceedings.068 